\title{
The Asterias Rubens Sea Star Igkappa Gene when Compared to Marthasterias Glacialis Sea Star Genome (Echinodermata)
}

\section{Michel Leclerc*}

Immunology of Invertebrates, Div : Biochem/Biology, Orléans University (France)

*Corresponding author: Michel Leclerc, 556 Immunology of Invertebrates, Div: Biochem/Biology, Orléans University (France)

Received date: 15 November, $2021 \mid$

Accepted date: 23 November, 2021 |

Published date: 26 November, 2021

Citation: Michel Leclerc. (2021) The Asterias Rubens Sea Star Igkappa Gene when Compared to Marthasterias Glacialis Sea Star Genome (Echinodermata). J Virol Viral Dis 1(1): doi https://doi.org/10.54289/JVVD2100105

Copyright: () 2021 Michel Leclerc. This is an open-access article distributed under the terms of the Creative Commons Attribution License, which permits unrestricted use, distribution, and reproduction in any medium, provided the original author and source are credited.

\section{Abstract}

The sea star IGKappa gene was cloned in 2014 by the use of primers. It was compared in the present work to Marthasterias glacialis sea star genome. A high identity was found with this last one.

\section{Introduction:}

The sequence of the sea star Asterias rubens IGKappa gene was described by our team, in 2014 [1]. Since we have tried to find homologies between this gene and genes from another Asterid: Marthasterias glacialis

We report, in the precedent paper, results obtained with these last ones by the use of blasts against human genes [2, 3].

The sequence of the sea star IGKappa gene is the following [1]:

5'GGATCCGGAGGAATGCGTGGCAACATGGCGTCTC TATGGATGTTCTTCTTTGTCGTGGGGATAACTTTAC
AACGGAGTTTGGCGATTTACACGTTTCGCGAGCAA CCGTCGGACACTAGCGCGTTGCAGGGGAGCACAGT GGTGCTTCACTGCTCCGTTGAGCAGTACATAAACA CCACGGCCATCGTTTGGTGGAGCCGTGACTCGGTC ATCAGCCACAACAAAGACCTGAAACTGTCCAGTCT AAACACCGACCAGCTCCAAAGGTACTCGATTTCAG GCGACGCATCTCGGGGGGAATTCAACCTTAAAATA GTGAACTTTACCGCCACAGACGCCGCCAGTTACCG CTGTCAGATGTAAGAATTC3'

Results:

Results are summarized in table below:

Blastn original sequence: Blastn results

Molecule type: dna

Query length: 357

\begin{tabular}{|c|c|c|c|c|c|c|c|c|}
\hline Description & Scientific name & $\begin{array}{l}\text { Max } \\
\text { score }\end{array}$ & $\begin{array}{l}\text { Total } \\
\text { score }\end{array}$ & $\begin{array}{l}\text { Query } \\
\text { cover }\end{array}$ & $\begin{array}{c}\text { E. } \\
\text { Value }\end{array}$ & $\begin{array}{l}\text { Per. } \\
\text { Ident }\end{array}$ & Acc Len & Accession \\
\hline $\begin{array}{c}\text { Predicted: Asterias Rubens } \\
\text { Uncharacterized Loc117296905 } \\
\text { (Loc117296905), mRNA }\end{array}$ & Asterias rubens & 634 & 634 & $97 \%$ & $2 \mathrm{e}-177$ & $99.43 \%$ & 4538 & XM_033780010.1 \\
\hline $\begin{array}{c}\text { Asterias Rubens Genome } \\
\text { Assembly, Chromosome: } 11\end{array}$ & Asterias rubens & 634 & 634 & $97 \%$ & $2 \mathrm{e}-177$ & $99.43 \%$ & 18069988 & LR699102.1 \\
\hline $\begin{array}{c}\text { Marthasterias Glacialis Genome } \\
\text { Assembly, Chromosome: } 6\end{array}$ & $\begin{array}{c}\text { Marthasterias } \\
\text { glacialis }\end{array}$ & 329 & 329 & $95 \%$ & $9 e-86$ & $84.16 \%$ & 28777708 & OU452224.1 \\
\hline
\end{tabular}

The graphic summary gives the opportunity to see the top3 Blast Hits: 


\section{Distribution of the top 3 Blast Hits on 3 subject sequences}

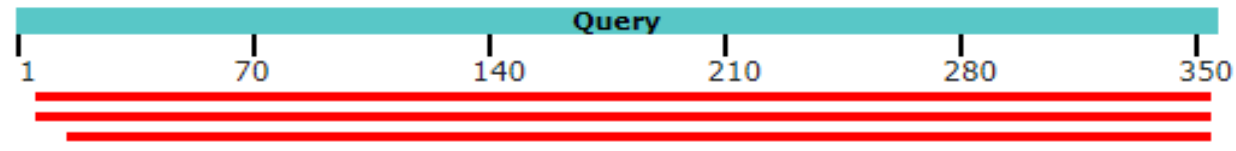

As for Alignements we find a high identity nearly $100 \%$ as shown below:

99\% Identities (347/349bp) with Predicted: Asterias Rubens Uncharacterized Loc117296905 (Loc117296905), mRNA

Reference Sequence: XM_033780010.1

Length: 4539

Aligment: 1546 - 1894

Query 7 to query 307 are resumed now with correspondence of nucleotids:

\begin{tabular}{|c|c|c|c|}
\hline uery & 7 & $\begin{array}{l}\text { ATGCGTGGCAACATGGCGTCTCTATGGATGTTCTTCTTTGTCGTGGGGATAACTTTACAA } \\
|||||||||||||||||||||||||||||||||||||||||||||||||||||||||||| \mid\end{array}$ & 66 \\
\hline bjct & 1546 & ATGCGTGGCAACATGTCGTCTCTATGGATGTTCTTCTTTGTCGTGGGGATAACTTTACAA & 1605 \\
\hline dery & 67 & $\begin{array}{l}\text { CGGAGTTTGGGATTACACGTTTCGCGAGCACCGTCGGCACTAGCGCGTTGCAGGGG } \\
\text { |||||||||||||||||||||||||||||||||||||||||||||||||||||||||||| }\end{array}$ & 126 \\
\hline bjet & 1606 & CGGAGTTTGGCGATTTACACGTTTCGCGAGCAACCGTCGGACACTAGCGCGTTGCAGGGG & 1665 \\
\hline uery & 127 & $\begin{array}{l}\text { AGCACAGTGGTGCTCACTGCTCCGTGAGCAGTACATAACACCACGGCATCGTTTGG } \\
|||||||||||||||||||||||||||||||||||||||||||||||||||||||||||||||||||||l|\end{array}$ & 186 \\
\hline ojct & 1666 & AGCACAGTGGTGCTTCACTGCTCCGTTGAGCAGTACATAAACACCACGGCCATCGTTTGG & 1725 \\
\hline uery & 187 & 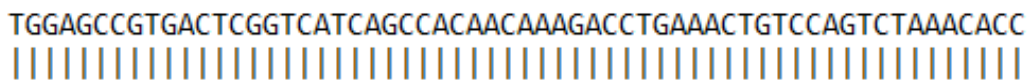 & 246 \\
\hline bjet & 1726 & TGGAGCCGTGACTCGGTCATCAGCCACAACAAAGACCTGAAACTGTCCAGTCTAAACACC & 1785 \\
\hline uery & 247 & $\begin{array}{l}\text { GACCAGCTCCAAAGGTACTCGATTTCAGGCGACGCATCTCGGGGGATTCAACCTTAAA } \\
\text { |||||||||||||||||||||||||||||||||||||||||||||||||||||||||||| }\end{array}$ & 306 \\
\hline bjct & 1786 & GACCAGCTCCAAAGGTACTCGATTTCAGGCGACGCATCTCGGGGGGAATTCAACCTTAGA & 845 \\
\hline uery & 307 & $\begin{array}{l}\text { ATAGTGAACTTTACCGCCACAGACGCCGCCAGTTACCGCTGTCAGATGT } 355 \\
||||||||||||||||||||||||||||||||||||||||||||||\end{array}$ & \\
\hline bjct & 1846 & ACAGACGCCGCCAGTTACCGCTGTCAGATGT & \\
\hline
\end{tabular}

\section{Conclusion:}

We retain from this bioinformatic analysis, a high identity between the Asterias rubens sea star IGKappa gene with the sea star Marthasterias glacialis genome. Those genes, neverthelessseem less evolved that the Ophuirid IGKappa gene we discovered 1 month ago [4] in terms of Immune functions.Sea stars belong to Asterid class. They are Echinodermasta like Ophuirids.

\section{References:}

1. Vincent N, et al. (2014) A new gene in A. rubens: A sea star Ig kappa gene. Meta Gene 2: 320-322.

2. Marchler-Bauer A, et al. (2017) CDD/SPARCLE: functional classification of proteins via subfamily domain architectures. Nucleic Acid Res 45(D): 200-203.

3. Marchler-Bauer A, et al. (2011) CDD: a Conserved Domain Database for the functional annotation of proteins. Nucleic Acid Res 39(D): 225-229.

4. Leclerc M (2021) E IG V-L K appa Expression from Sea Star Igkappa Gene. J Clin Class Immunol. 1(1). 